EPJ Web of Conferences 19, 07009 (2012)

DOI: $10.1051 /$ epjconf/20121907009

(C) Owned by the authors, published by EDP Sciences, 2012

\title{
The dynamics of long-lived spiral arms
}

\author{
M.S. Fujii ${ }^{1, a}$, J. Baba², T.R. Saitoh ${ }^{2}$, E. Kokubo ${ }^{3}$, J. Makino² and K. Wada ${ }^{1}$ \\ ${ }^{1}$ Graduate School of Science and Engineering, Kagoshima University, Japan \\ ${ }^{2}$ Interactive Research Center of Science, Tokyo Institute of Technology, Japan \\ ${ }^{3}$ Division of Theoretical Astronomy, National Astronomical Observatory of Japan, Japan
}

\begin{abstract}
It has been believed that spiral arms in pure stellar disks decay in several galactic rotations due to the heating by the spiral arms. However, it might be caused by a numerical heating. We performed a three-dimensional $N$-body simulations with a sufficiently large number of particles and found that stellar disks can maintain spiral arms for more than $10 \mathrm{Gyr}$ without the help of cooling. Spiral arms are transient and recurrent and they heat disk with a heating rate, $d Q / d t$, correlated to the spiral amplitude $\left|A_{m}\right|$. On the other hand, $\left|A_{m}\right|$ is suppressed by Toomre's $Q$. Therefore, the dynamical heating becomes less effective in the later phase of the evolution. This mechanism maintain the spiral arms for more than $10 \mathrm{Gyr}$.
\end{abstract}

\section{INTRODUCTION}

Multiple spiral arms in a galactic disk develop due to swing amplification and they are transient and recurrent [9]. Since the simulation of stellar disks with multiple spiral arms performed by Sellwood \& Carlberg (1984), it has been believed that spiral arms in pure stellar disks decay in several galactic rotations due to the increase of the velocity dispersion [8]. Therefore, some cooling mechanism due to the gas and star formation have been investigated $[1,2,8]$. On the other hand, pure $N$-body simulations with three-dimensional and a large number of particle have not been performed. Such a simulation is important to avoid artificial heating by the two-body relaxation, which might have significantly enhanced the decay of spiral arms.

\section{2. $N$-BODY SIMULATIONS}

We performed a series of $N$-body simulations of stellar disks in a fixed spherical dark halo potential. We adopted the NFW model [7] as the dark halo model with the concentration parameter of the halo, $c=10$. The virial radius of halo, $R_{\mathrm{h}}$, is $122 \mathrm{kpc}$, and the mass within $R_{\mathrm{h}}, M_{\mathrm{h}}$ is $6.4 \times 10^{11} M_{\odot}$. We adopted an exponential disk model as disk models with the scale radius, $R_{\mathrm{d}}$, of $3.4 \mathrm{kpc}$ and the scale height, $z_{\mathrm{d}}$ of $0.34 \mathrm{kpc}$. We performed simulations with five different resolutions, $N=3 \times 10^{7}, 9 \times 10^{6}$, $3 \times 10^{6}, 1 \times 10^{6}$, and $3 \times 10^{5}$ (hereafter, $30 \mathrm{M}, 9 \mathrm{M}, 3 \mathrm{M}, 1 \mathrm{M}$, and $300 \mathrm{k}$, respectively). We generated initial disk models using the Hernquist method [3]. We used a Burnes-Hut treecode on GRAPE-7 [4] and GRAPE-DR [6] with the opening angle, $\theta$, of 0.4 and with the center-of-mass approximation [5]. For the time integration, we used a leapfrog integrator with a fixed stepsize of $\Delta t=0.29 \mathrm{Myr}$ for $N=30 \mathrm{M}$, $9 \mathrm{M}$, and $1 \mathrm{M}$ models and $\Delta t=0.15 \mathrm{Myr}$ for the other models. The gravitational potential is softened using Plummer softening of $30 \mathrm{pc}$.

\footnotetext{
${ }^{a}$ e-mail: fujii@cfca.jp
}

This is an Open Access article distributed under the terms of the Creative Commons Attribution-Noncommercial License 3.0, which permits unrestricted use, distribution, and reproduction in any noncommercial medium, provided the original work is properly cited. 

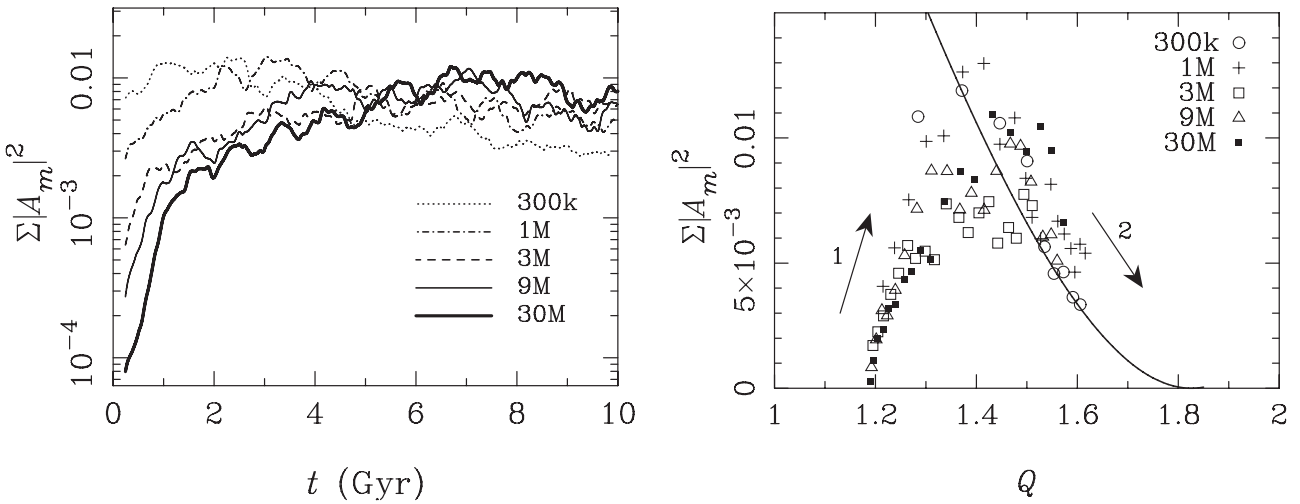

Figure 1. Left: time evolution of total power, $\sigma_{m=1}^{10}\left|A_{m}\right|^{2}$. Right: relation between $Q$ and total power. Arrows show the direction of the time evolution. Black curve shows $\left(0.1 A_{m}\right)^{2}$.

\section{LONG-LIVED SPIRAL ARMS}

Spiral arms developed from Poisson noise and they heat up the disk by scattering the disk stars. We found that the heating rate, $d Q / d t$, correlates with the squared Fourier amplitude of spiral arms, $\left|A_{m}\right|^{2}$. While the spiral arms heat the disk, they survived more than $10 \mathrm{Gyr}$ when the number of particles is large enough $\left(N>3 \times 10^{6}\right)$. Left panel of figure 1 shows the evolution of the total power of the spiral arms (the sum of the squared Fourier amplitudes). We also found that the spiral amplitude is limited by Toomre's $Q$ (see right panel of figure 1). Therefore, the heating rate slows down as the disk heats up. This self-regulating mechanism maintains spiral arms. In the case of small $N$, on the other hand, the spiral arms reached a higher amplitude in the beginning of the simulation. It causes a rapid heating, and as a result, the spiral arms disappear in several Gyr.

\section{SUMMARY}

We performed three-dimensional $N$-body simulations of pure stellar disks with spiral arms and investigated their dynamical evolution. Contrary to previous results, we found that spiral arms in pure stellar disks can survive for more than $10 \mathrm{Gyr}$, when we use a sufficiently large number of particles $\left(N>3 \times 10^{6}\right)$. We also found that spiral arms of a stellar disk are self-regulated. The spiral arms grow by the swing amplification up to their maximum amplitudes determined by Toomre's $Q$ value at the moment. The spiral arms heat up the disk, or increase the velocity dispersion of stars, by scattering the disk stars. As a result, $Q$ increases, and the amplitude of spirals is suppressed. This self-regulating relation among $Q$, spiral amplitudes, and the heating rate maintains the spiral arms for more than $10 \mathrm{Gyr}$.

\section{References}

[1] Bottema, R., MNRAS, 344, (2003) 358

[2] Elmegreen, B. G., \& Thomasson, M., A\&A, 272, (1993) 37

[3] Hernquist, L., ApJS, 86, (1993) 389

[4] Kawai, A., Fukushige, T., \& Makino, J., Proc. 2006 ACM/IEEE Conf. on Supercomputing, (2006)

[5] Makino, J., PASJ, 56, (2004) 521

[6] Makino, J., Hiraki, K., \& Inaba, M., Proc. 2007 ACM/IEEE Conf. on Supercomputing, (2007)

[7] Navarro, J. F., Frenk, C. S., \& White, S. D. M., ApJ, 490, (1997) 493

[8] Sellwood, J. A., \& Carlberg, R. G., ApJ, 282, (1984) 61

[9] Toomre, A., Structure and Evolution of Normal Galaxies (Cambridge University Press,1981) 111 